\title{
Comment on "Venous thrombosis: who should be screened for thrombophilia in 2014?"
}

To the Editor We read the review article by Paul A. Kyrle with great interest. ${ }^{1}$ We fully agree with the author that systematic indiscriminate laboratory thrombophilia screening in all patients with the first unprovoked venous thromboembolic episode (VTE) is unwarranted and should be discouraged. However, there is still ${ }^{1}$ one important and unanswered question: who should be screened for thrombophilia? We would all probably agree that owing to age dependency of VTE, elder patients should not be screened. However, those aged from 45 to 50 years (or even younger) with unprovoked episodes certainly require our attention. At this point, some differences regarding inherited and acquired thrombophilia may be of issue.

The utility of laboratory investigation for inherited thrombophilia in patients with VTE and their asymptomatic relatives has been largely debated, leading to the production of several guidelines by scientific societies and various working groups. As the risk largely depends on the family history of VTE, the identification of asymptomatic carrier among relatives of the probands with VTE and thrombophilia could reduce cases of provoked VTE by offering them primary antithrombotic prophylaxis during risk situations. In most guidelines, this is considered justified only for relatives of probands with a deficiency of natural anticoagulants or multiple abnormalities. Counselling asymptomatic female relatives of individuals with VTE and/or thrombophilia before planned conception or treatment with hormonal preparations should also be recommended depending on the risk driven by the type of thrombophilia and the family history of VTE. ${ }^{2}$

Acquired thrombophilia mainly involves testing for antiphospholipid antibodies necessary to establish the diagnosis of antiphospholipid syndrome. Even if evidence on the risk of recurrent thrombosis in patients with antiphospholipid antibodies is low or available data are of poor quality, we still believe the testing may be warranted. The tested group should include: younger patients ( $<50$ years), patients with a systemic autoimmune disease and any VTE episode in history, patients with accidentally found prolonged activated partial thromboplastin time (after exclusion of clotting factor deficiency, mainly FXII), and women with recurrent spontaneous pregnancy loss. ${ }^{3}$ The latest clinical guidelines of the National Institute for Health and Care Excellence also recommend testing for antiphospholipid antibodies: in patients who have had unprovoked deep vein thrombosis (DVT) or pulmonary embolism (PE; if withdrawal of anticoagulation treatment is planned); and in patients who have had unprovoked DVT or PE and have a first-degree relative with previous DVT or PE. ${ }^{4}$

In addition, we would not lightly discard the option to treat positive (especially so called "triple positive") patients indefinitely in the light of recent data showing that, in patients diagnosed with antiphospholipid syndrome during the 10 -year period, $36.5 \%$ of the fatal cases were related to severe thrombosis. ${ }^{5}$

We hope this additional information will help readers of the Polish Archives of Internal Medicine decide whether thrombophilia testing may be of value for primary and secondary VTE prophylaxis in their patients.

Author names and affiliations Krystyna Zawilska, Jacek Musiał; members of the Working Group on Haemostasis of the Polish Society on Haemtology and Transfusion Medicine (K.Z.: Poznan University of Medical Sciences, Center for Diagnosis and Therapy, INTERLAB, Poznań, Poland; J.M.: 2nd Department of Internal Medicine, Jagiellonian University Medical College, Kraków, Poland)

Corresponding author Prof. Jacek Musiał, MD, $\mathrm{PhD}$, II Katedra Chorób Wewnętrznych, Uniwersytet Jagielloński, Collegium Medicum, ul. Skawińska 8, 31-066 Kraków, phone: +48-12-430-53-14, fax: +48-12-430-50-66, e-mail: mmmusia@cyf-kr.edu.pl

Conflict of interest The authors declare no conflict of interest.

\section{REFERENCES}

1 Kyrle PA. Venous thrombosis: who should be screened for thrombophilia in 2014? Pol Arch Med Wewn. 2014; 124: 65-69.

2 De Stefano V, Rossi E. Testing for inherited thrombophilia and consequences for antithrombotic prophylaxis in patients with venous 
thromboembolism and their relatives. A review of the Guidelines from Scientific Societies and Working Groups. Thromb Haemost. 2013; 110: 697-705.

3 Pengo V, Tripodi A, Reber G, et al. Update of the guidelines for lupus anticoagulant detection. J Thromb Haemost. 2009; 7: 1737-1740.

4 Venous thromboembolic diseases: the management of venous thromboembolic diseases and the role of thrombophilia testing. NICE clinical guidelines; issued: June 2012:CG144. http//guidance.nice.org.uk. Accessed January 2014

5 Cerevera R, Serrano R, Pons- Estel GJ, et al Morbidity and mortality in the antiphospholipid syndrome during a 10-year period: a multicenter prospective study of 1000 patients. Ann Rheum Dis. 2014. doi: 10.1136/annrheumdis-2013-204838. 